\title{
Geschlechter- und intersektionalitätskritische Perspektiven auf Konzepte der Softwaregestaltung
}

\author{
Tanja Paulitz, Bianca Prietl \\ Institut für Soziologie, Rheinisch-Westfälische Technische Hochschule Aachen
}

\section{Die Vergeschlechtlichung informatischer Artefakte und Geschlechtertheorien}

Frauen- und Geschlechterforschung zur Informatik ist seit jeher ein zentraler Strang der feministischen Technikforschung und hat eine mittlerweile beachtliche Menge an Forschungen hervorgebracht (zum Überblick siehe u. a. Bath, Schelhowe und Wiesner, 2010; Schelhowe, 2006); u. a. beschäftigen sich Untersuchungen mit der Vergeschlechtlichung von informatischen Artefakten wie Software und anderen Informationstechnologien. Diese Studien zeigen, wie alltägliche Vorstellungen von Geschlecht ebenso wie strukturelle Ungleichheiten im Geschlechterverhältnis in die Produkte der Informatik eingeschrieben und durch diese (re-)produziert werden (Bowker und Star, 2000; Crutzen, 2003; Hofmann, 1999; John, 2006; Lübke, 2005; Rommes, 2002, 2014; Rommes, van Oost und Oudshoorn, 1999). Eine zentrale Rolle kommt dabei der sogenannten „I-Methodology“ zu (Bath, 2009, S. 99-100). Die „I-Methodology“ gilt als eine der meist eingesetzten Gestaltungstechniken im Bereich der Informations- und Kommunikationstechnologien. Sie bezeichnet ein Vorgehen, bei dem die Entwickler_innen sich selbst als typische Nutzer_innen imaginieren und so Designentscheidungen auf Basis ihrer eigenen Präferenzen treffen; es ist dieser subjektive Charakter, auf den die Bezeichnung „I-Methodology“ hinweist. Als problematisch wird sie von feministischer Seite v. a. deshalb angesehen, weil die strukturellen Arbeitsverhältnisse in der Softwareentwicklung stark von Männern dominiert sind, sodass etwa heterogene Gruppen von Nutzer_innen nicht mitgedacht sind. Darüber hinaus haben Softwareentwickler_innen ein besonderes Verhältnis zu Technik und Kompetenzen im Umgang mit Technik, die nicht als repräsentativ für die Gesamtgesellschaft angesehen werden können (Rommes, 2014, S. 4648).

In den letzten Jahren gab es verstärkt Initiativen, Geschlechterperspektiven gezielt in die natur- und technikwissenschaftliche Forschung sowie Technikentwicklung einzubringen (siehe für eine aktuelle kritische Diskussion Ernst und Horwath, 2014a). Ein durchaus prominenter Strang zielt darauf ab, gerade die Verschiedenheiten von möglichen Nutzer_innen in der Softwareentwicklung zu berücksichtigen und so Gestaltungsvorgaben im Hinblick auf Bedürfnisse von Frauen und Männern zu diversifizieren. Zu nennen ist in diesem Zusammenhang das von der Fraunhofer Gesellschaft geführte Projekt mit dem Titel „Discover Gender" sowie das jüngst unter der Leitung der amerikanischen Wissenschaftsforscherin 
Londa Schiebinger durchgeführte und von Europäischer Union und der U.S. National Science Foundation unterstützte Projekt „Gendered Innovations“ (Schiebinger, Klinge, Sánchez de Madariaga, Schraudner und Stefanick, 2011-2013). Zu derartigen Versuchen, die „I-Methodology“ zu verlassen und Technikentwicklung zu diversifizieren, wurde kritisch angemerkt, dass dabei der sozialen Konstruiertheit von Geschlecht nur unzureichend Rechnung getragen werde (Paulitz, 2008, S. 783); konkret heißt das, dass die Analyse und Integration von Geschlechteraspekten weniger auf Geschlechtsunterschiede fokussieren sollte, um gerade nicht Gefahr zu laufen, alltägliche Vorstellungen über Frauen und Männer zu reproduzieren. Vielmehr sei es erforderlich, gerade den vielfältigeren Bedürfnissen und Lebenslagen von Menschen aus verschiedensten sozialen Gruppen sowie Interdependenzen zwischen der Kategorie Geschlecht und anderen sozialen Ungleichheitskategorien wie Alter oder Herkunft mehr Beachtung zu schenken (Bath, 2007, S. 4). In eine ähnliche Richtung zielen die Ergebnisse des europaweiten Forschungsprojektes SIGIS (Strategies of Inclusion; Gender in the Information Society), das empirisch die Praktiken der Technikentwicklung von Unternehmen untersucht hat, die das Ziel verfolgten, geschlechterinklusive Informations- und Kommunikationstechnologien zu entwickeln. In der Praxis hieß das zumeist, dass geschlechterstereotype Vorstellungen der Entwicklungsarbeit zugrundegelegt wurden und somit eine stark verkürzte Sicht auf Geschlecht realisiert wurde (Rommes, 2013).

Vor diesem Hintergrund zielt dieser Beitrag auf Bedarf und Möglichkeiten der Weiterentwicklung vorhandener Methodik der Softwareentwicklung aus geschlechter- und intersektionalitätskritischer Perspektive und rückt dabei eine methodologisch-epistemologische Betrachtung ins Zentrum. Damit versteht er sich als Beitrag zu einer grundlagenorientierten Diskussion aus Sicht der Geschlechterforschung und beansprucht nicht, die tatsächliche Praxis der Softwareentwicklung zu rekonstruieren oder evaluieren. Hierfür knüpfen wir maßgeblich an theoretische Einsichten der Geschlechterforschung an, die Geschlecht grundsätzlich als soziale Konstruktion betrachten (Helduser, Marx, Paulitz und Pühl, 2004). Das heißt, wir gehen nicht davon aus, dass es sich bei Männern und Frauen einfach um zwei klar zu unterscheidende soziale Gruppen mit eindeutig benennbaren, unterschiedlichen Vorlieben, Bedürfnissen und Fähigkeiten handelt. Hingegen stehen die gesellschaftlichen (Konstruktions-)Prozesse im Mittelpunkt, die erst solche sozialen Gruppen, Vorlieben, Bedürfnisse und Fähigkeiten erzeugen bzw. letztere Frauen und Männern zuschreiben. Hiervon ausgehend, beziehen wir im Folgenden Geschlecht in drei verschiedenen Hinsichten in unsere Betrachtung mit ein:

a) Geschlecht als gesellschaftliche Strukturkategorie (Becker-Schmidt, 1987; Beer, 1990; Knapp, 1990) zu betrachten, lenkt den Blick darauf, wie Frauen und Männern in der Gesellschaft unterschiedliche Tätigkeitsfelder zugewiesen werden, die in der Regel hierarchisch angeordnet sind; beispielhaft zu nennen sind hier die Trennung und Ungleichbewertung von Erwerbs- und Familienarbeit oder die strukturelle Ungleichverteilung von Frauen und Männern auf unterschiedliche (und unterschiedlich gesellschaftlich anerkannte) Berufe wie Ingenieur- und Pflegeberufe.

b) In Form von Wissen über die Unterschiede zwischen Frauen und Männern ist Geschlecht tief in alltäglichen, üblicherweise unhinterfragten Alltagsvorstellungen verankert und wird im alltäglichen Handeln immer wieder reproduziert (Gildemeister, 2008; Wetterer, 2008). Bedeutsam an diesen Alltagsvorstellungen ist auch, dass sie die Geschlechterdifferenz als „natürlich“ betrachten und unterschiedliche Lebenslagen innerhalb der Gruppe der Frauen bzw. innerhalb der Gruppe der Männer tendenziell ausblenden. Solche in die tieferen 
Schichten unseres Wissens sedimentierten kulturellen Geschlechtersymboliken und ihre Bedeutung für naturwissenschaftliche Erkenntnisgewinnung wie technikwissenschaftliche Artefaktgestaltung wurden in den vergangenen Jahren insbesondere von einer epistemologisch-wissenschaftskritisch argumentierenden Geschlechterforschung zum Gegenstand der Analyse gemacht (siehe zum Überblick u. a. Lucht und Paulitz, 2008; Singer, 2005).

c) Aus intersektionaler Perspektive sind stereotype Vorstellungen von zwei Geschlechtern auch deshalb aufzubrechen, weil neben Geschlecht weitere soziale Ungleichheiten bedeutsam sind, die in den auf klare Differenz fokussierten Alltagsvorstellungen zunächst nicht mit berücksichtigt sind. So existieren nicht nur weitgehend unhinterfragte Alltagsklischées in Bezug auf Frauen und Männer, sondern auch in Bezug auf kulturelle Zugehörigkeiten, sexuelle Orientierung, Religion, soziale Schichten, Behinderung usw., die in eine kritische Analyse des Alltagswissens mit einbezogen werden sollten. Was wird also beispielsweise an Alltagswissen in der Softwareentwicklung eingesetzt, wenn man als potentielle_n Nutzer_in einen Arbeitslosen mit deutscher Staatsangehörigkeit, eine muslimische Dozentin im Alphabetisierungskurs, eine 30jährige Finanzbrokerin, einen Versicherungsmakler kurz vor der Rente oder eine Leiterin eines Selbsthilfezentrums mit fortgeschrittener Multipler Sklerose im Blick hat? Solche Perspektiven wurden in der jüngeren Vergangenheit in der Geschlechterforschung unter dem Stichwort „Intersektionalität“ diskutiert (siehe u. a. Kerner, 2009; Smykalla und Vinz, 2013; Winker und Degele, 2009). Dabei wurde hervorgehoben, dass sich soziale Lagen von Frauen hochgradig unterscheiden können, aber auch, dass alltägliche Vorstellungen von Menschen komplexe, interdependente und oftmals unhinterfragte Konstruktionen darstellen. So mag sich die alltägliche Vorstellung von Weiblichkeit in Abhängigkeit davon stark unterscheiden, ob sie sich auf eine Muslima, eine Frau mit Behinderung oder eine Mehrheitsdeutsche bezieht.

Mit Blick auf Informations- und Kommunikationstechnologien lässt sich Technikentwicklung grundsätzlich als Teil sozialer Konstruktionsprozesse von Geschlecht (und ihrer Interdependenz mit anderen sozialen Ungleichheitskategorien) begreifen, in deren Zuge, wie eingangs ausgeführt, Zuschreibungen und stereotype Annahmen unmittelbar in der Gestaltung der Artefakte materialisiert werden. Dies ist zum einen Effekt ungleicher struktureller (Arbeits-) Verhältnisse, zum anderen Effekt von unhinterfragtem Alltagswissen über die zukünftigen Nutzer_innen, typische Human-Computer Interaction (HCI) und die vermeintlich eindeutigen Techniknutzungsbedürfnisse und -voraussetzungen von Nutzer_innen. Ausgehend davon erscheint es erforderlich, solche strukturellen Hierarchien ebenso wie die alltäglichen Vorstellungen von Menschen zu hinterfragen und methodische Ansätze der Softwareentwicklung mit entsprechend reflexiven Komponenten auszustatten. Wie Ernst und Horwath (2014b) indessen zu Recht festhalten, mag es keinen ,'one best way' for a feminist design of new technologies“ (S. 10) geben. Dennoch folgt die vorliegende geschlechter- und intersektionalitätskritische Arbeit in Anlehnung an diese Autorinnen dem grundsätzlichen Vorschlag, „to take gender into account in a theoretically reflected and methodologically systematic way in order to counteract problematic gendering“ (S. 10). Der vorliegende Beitrag dient der exemplarischen Sondierung eines solchen Vorhabens und wendet sich hierfür den für diese Unternehmung besonders anschlussfähigen partizipativen Methoden der Softwareentwicklung zu, Methoden also, die Software unter Beteiligung von Nutzer_innen entwerfen und erproben. Wie Buchmüller (2013) gezeigt hat, sind partizipative Designmethoden nicht als zwingend geschlechtergerechter oder sozial fairer anzusehen. Ohne systematische feministisch orientierte Reflexion würden sie vielmehr bestehende Ungleichheitsverhältnisse repro- 
duzieren. Eingedenk dieser kritischen Haltung, fokussiert dieser Beitrag auf Methoden der Gestaltung von Human-Computer Interaction.

Dazu wird anhand einer konkreten Methodik - nämlich szenarienbasierter Ansätze - exemplarisch beleuchtet, wo und wie die o. g., vorwiegend in der sozial- und kulturwissenschaftlichen Geschlechterforschung diskutierten Perspektiven, mit bestehenden Methodiken der Gestaltung von HCI verbunden werden können. Szenarienbasierte Ansätze verstehen sich, folgt man Bath (2009), als eine von vielen Methoden, die im Rahmen einer geschlechterkritischen Entwicklung informatischer Artefakte zum Einsatz kommen können (S. 233 ff.). Grundsätzliches Ziel szenarienbasierter Ansätze ist die nutzer_innen-zentrierte Gestaltung von HCI auf Basis von Techniknutzungsszenarien, die die Form von Geschichten über den konkreten Nutzungszusammenhang haben. Diese Geschichten werden in wiederholter Auseinandersetzung mit den (potentiellen) Nutzer_innen und Kund_innen im Laufe des Softwareentwicklungsprozesses entworfen und mehrfach reformuliert. Die damit angestrebte intensive Auseinandersetzung mit dem Nutzungskontext und seinen Akteur_innen folgt eben dem Anliegen, von stereotypen Annahmen über Nutzer_innen, ihre Vorlieben und Kompetenzen Abstand zu nehmen. Insofern können szenarienbasierte Ansätze als besonders reflexive und ambitionierte Methode im Bereich von HCI und Usability angesehen werden, um durch systematischen Einbezug der Nutzungsseite von der viel kritisierten „I-Methodology“ weg zu kommen. Auch deshalb eignen sie sich für eine geschlechter- und intersektionalitätskritische Weiterentwicklung.

Die hier zur Diskussion gestellte Weiterentwicklung szenarienbasierter Ansätze der Softwareentwicklung setzt primär an zwei Stellen an: einmal an methodischen Überlegungen zur Erhebung und Erstellung der Geschichten und Szenarien, und einmal an der methodologischepistemologischen Reflexion der Inhalte dieser Geschichten und Szenarien, in der strukturelle Hierarchien und alltägliche Vorstellungen von Geschlecht im Zentrum stehen. Um Perspektiven im Sinne der hier eingebrachten Geschlechter- und Intersektionalitätsforschung zu eröffnen, wird eine interdisziplinäre Zusammenarbeit im Rahmen der partizipativ szenarienbasierten Softwareentwicklungsprozesse vorgeschlagen. Damit schließt unser Vorschlag an das Argument von Corinna Bath an, wonach eine komplexe und den Einsichten der Frauenund Geschlechter- sowie Technikforschung Rechnung tragende Integration von Geschlechteraspekten in die Technikentwicklung ,einer intensiven interdisziplinären Übersetzungsund Zusammenarbeit“" (2007, S. 5) bedürfe.

Dazu werden nun im Folgenden szenarienbasierte Ansätze der Softwaregestaltung näher beschrieben, auf ihren Weiterentwicklungsbedarf im Sinne der hier eingenommenen Perspektive ausgeleuchtet und exemplarisch in Hinblick auf ihr Potential diskutiert. Im abschließenden Fazit wird eine Perspektive auf eine gesellschaftsverändernde Softwaregestaltung eröffnet.

\section{Weiterentwicklungspotentiale von szenarienbasierter Softwaregestaltung}

Szenarienbasierte Ansätze gewannen in der Softwareentwicklung, vor allem im Bereich „Human-Computer Interaction“, als nutzer_innen-zentrierte Methode im Lauf der 1990er Jahre an Bedeutung (Rosson und Carroll, 2002): „SEP [Scenario-based Engineering Process; $\mathrm{TP} \& \mathrm{BP}]$ is a user-centered methodology for systems or business process engineering that 
employs the use of scenarios to scope, bound, and focus analysis, design, development, and evaluation activities“ (McGraw und Harbison, 1997, S. 8-9).

Das Kernelement dieses Softwareentwicklungsansatzes, ein „Szenario“, ist in seiner Grundform eine Geschichte, die erzählt, wie eine oder mehrere Person(en) eine oder mehrere (Arbeits-)Tätigkeit(en) ausführen. Die Geschichte soll auf diese Weise die konkrete Situation in Szene setzen, in der die Tätigkeit stattfindet, die Erfahrungen, Verhaltensweisen und Interessen der betreffenden Person(en) sowie deren Überlegungen in Hinblick auf mögliche Durchführungsweisen und die damit verbundenen Tätigkeitsziele. „SEP focuses on what potential system users or key performers actually do in the workplace" (McGraw und Harbison, 1997, S. 11; Hervorhebungen im Original). Die zentrale Idee dabei ist, dass ein Szenario die Aufmerksamkeit auf die Personen und ihre Tätigkeiten richtet: „Scenarios focus designers on the needs and concerns of people in the real world" (Rosson und Carroll, 2002, S. 22). Szenarien können in vielfältiger Weise dargestellt werden: „through video clips, drawings, timelines, text outlines, simulations, animations, event traces, and process diagrams“" (McGraw und Harbison, 1997, S. 38). Derartige Geschichten über konkrete Techniknutzungen könnten - so die Idee - zu jedem Zeitpunkt der Softwareentwicklung entworfen und diskutiert werden. So lassen sie sich beispielsweise als „Problemszenario“ dazu einsetzen, die Ergebnisse einer Anforderungsanalyse darzustellen oder als „Interaktionsszenario“, um einen Designvorschlag auszuformulieren und $\mathrm{zu}$ veranschaulichen. Kostengünstig und aufwendungsgering sind sie in einem iterativ-zyklischen Softwareentwicklungsprozess jederzeit leicht anzufertigen und ebenso leicht abzuändern. Als weiterer Vorteil von Szenarien wird genannt, dass sie in einer Sprache verfasst sind, die ein jeder und eine jede versteht, sodass sie die Kommunikation zwischen Entwickler_innen, Nutzer_innen und weiteren Interessensgruppen erleichtern und die Entwicklungsprozesse auf diese Weise inklusiver gestalten, denn ,[a]11 project members can 'speak' the language of scenarios" (Rosson und Carroll, 2002, S. 23). Aus diesem Grund seien szenarienbasierte Ansätze auch besonders geeignet für partizipative Technikgestaltung. Zentral ist dabei stets die Erhebung der Situation, in der die Techniknutzung stattfindet oder stattfinden soll. Als mögliche Erhebungsmethoden werden u. a. Interviews, Beobachtungen, Arbeitsprozess- oder Aufgabenanalysen, Gruppenarbeiten wie Fokusgruppen oder Brainstorming sowie Dokumentenanalysen genannt (McGraw und Harbison, 1997, S. 33).

Obgleich politische Ziele nicht dezidiert mit der Szenarien-Technik verbunden sind, ist darauf hinzuweisen, dass Rosson und Carroll (2002) am Ende ihres Lehrbuches unter „Ethics of Usability“ Fragen sozialer Ungleichheit im Zusammenhang mit Zugang zu und Nutzung von informatischen Artefakte zumindest kursorisch ansprechen. Insbesondere wird auf die speziellen Bedürfnisse von Personen mit Behinderung sowie von älteren Personen hingewiesen, auf die in der Technikgestaltung Rücksicht genommen werden müsse (vgl. S. 356-358). Dieser selektive Verweis kann als Indiz dafür gewertet werden, dass mögliche Ungleichheiten in der Human-Computer Interaction zwar angedacht, aber bislang noch nicht systematisch in die Softwareentwicklung einbezogen werden. Dazu wurde bereits kritisch angemerkt, dass auf diese Weise das Potential szenarienbasierter Ansätze, die in der „I-Methodology“ liegende Problematik der Reproduktion von Stereotypen zu überwinden, nicht ausgeschöpft wird (Bath, 2009, S. 233 ff.).

Insofern zeigt sich, dass szenarienbasierte Ansätze einen Weiterentwicklungsbedarf in Richtung der Integration von Perspektiven sozialer Ungleichheit aufweisen, um ihrem eigenen Anspruch, für bestimmte Nutzungskontexte bedarfsorientierte und nutzbare Software zu 
entwickeln, besser gerecht zu werden. Im Folgenden wird es daher darum gehen, dem damit diagnostizierten prinzipiellen Weiterentwicklungsbedarf detaillierter auf die Spur zu kommen und so Softwaregestaltungsprozesse ,um grundsätzliche, wissenschaftskritische, politische und feministische Perspektiven“ (Bath, 2009, S. 237) zu ergänzen.

Hierfür werden wir zwei Aspekte herausgreifen, die aus der eingangs erläuterten theoretischen geschlechter- und intersektionalitätskritischen Sicht vielversprechende Richtungen der Weiterentwicklung wären: der Einsatz von offenen Instrumenten der Erhebung von Szenarien einerseits und die kritische Reflexion auf die Inhalte der den Szenarien zugrundeliegenden Geschichten andererseits.

\section{$2.1 \quad$ Offene Erhebungsmethodik}

Es wurde bereits erwähnt, dass in den einschlägigen Lehrbüchern szenarienbasierter Ansätze eine Bandbreite an sozialwissenschaftlichen Erhebungsmethoden vorgeschlagen wird, um die für die jeweilige Aufgabe relevanten Szenarien zu ermitteln. Neben Interviews kommen auch Beobachtungen, Dokumentenanalysen und vieles mehr zum Einsatz. Allerdings werden gerade diese nicht-standardisierten methodischen Zugänge als nachteilig betrachtet, da sie Informationen zumeist nicht in strukturierter Form und häufig quer zur Denklogik der Entwickler_innen zugänglich machen: „Disadvantages of the interview relate primarily to its open, communicative nature." (McGraw und Harbison, 1997, S. 207) Bemängelt wird die offene Kommunikationssituation, in der Kontrollverlust ebenso droht wie das Abweichen von der ,Zielgeraden“, auf die das Projekt zusteuern sollte. McGraw und Harbison empfehlen den Entwickler_innen daher steuernd einzugreifen, um Kontrolle und Führung zurück zu gewinnen und so die Projektziele nicht zu gefährden: „If the interview takes an unwanted detour, we can point to the agenda to help us regain control $[\ldots]$. The analyst will use leadership and interview management techniques to keep the interview body on track, progressing toward the goals." (1997, S. 182). Betrachtet man diese Vorgehensweise kritisch, so stellt sich indessen die Frage, um welchen Preis diese methodische Kontrolle ausgeübt wird. Wird hier von einer klaren Zielvorgabe ausgegangen, gibt es einen eindeutigen „,track“, zu dessen Wahrung die Eigenlogik der befragten Nutzer_innen begrenzt werden muss? Es ist auf Basis der hier zitierten, allgemeinen methodischen Empfehlung nicht zu klären, wie eng oder weit jeweils die Zielvorgabe eines Projektes ist, von der nicht abgewichen werden soll. Doch liegt genau hier ein Eingriffspunkt, an dem über den Grad der Offenheit nachgedacht werden muss, will man nicht doch - und zwar vermittels einer im Erhebungsverfahren angelegten Begrenzung - frühzeitig wertvolle Nutzer_innenperspektiven ausschließen. So besteht aus der Perspektive sozialer Ungleichheiten ja gerade die Möglichkeit, dass Softwareentwickler_innen genau dort im Interview die Führung übernehmen, wo von der „I-Methodology“ abgewichen wird, wo Nutzer_innen sperrige Geschichten entwickeln, in denen etwa strukturelle Widersprüche im gesellschaftlichen Geschlechterverhältnis zur Sprache kommen oder dem gängigen Alltagswissen zuwiderlaufende Geschichten entworfen werden.

Um diesen Engführungen zu entgehen und tragfähige Szenarien zu entwickeln, könnte eine partizipative, szenarienbasierte Methodik zukünftig hingegen die Interaktion im Erhebungsprozess systematisch reflektieren und sich methodisch-geleitet auf die Spur der Befragten einlassen. Produktive Anregungen bieten u. E. qualitative Erhebungsverfahren, die eben dem Prinzip der Offenheit folgen und hieraus gerade die Stärken ihrer Informationsgewinnung beziehen. Das Prinzip der Offenheit besagt, so die Pionierformulierung von Hoffmann-Riem, 
„dass die theoretische Strukturierung des Forschungsgegenstandes zurückgestellt wird, bis sich die Strukturierung des Forschungsgegenstandes durch die Forschungssubjekte [die Befragten] herausgebildet hat" (1980, S. 343). Ziel ist die Rekonstruktion von bislang gerade nicht bekannten Ordnungsstrukturen, über die die Befragten etwas erzählen können. So entsteht die Chance, eng am Kontext die „Lebenswelten ,von innen heraus“ aus der Sicht der handelnden Menschen zu beschreiben“ (Flick, Kardorff und Steinke, 2007, S. 14). Für die geschlechter- und intersektionalitätskritische Weiterentwicklung partizipativer Softwareentwicklung beinhaltet eine methodische Orientierung am Prinzip der Offenheit daher das Potential, gerade den strukturellen Widersprüchen und gegebenenfalls quer liegenden Konstruktionen von Geschlecht auf die Spur zu kommen und sie in die Szenarien-Arbeit einzubeziehen. Hier entstehen auf diese Weise Öffnungen für diejenigen, die in der Regel eben nicht mitgedacht sind oder deren Lebenslagen aufgrund struktureller Ausgrenzungen tendenziell in Nutzungsszenarien ausgeschlossen bleiben.

\subsection{Reflexive Arbeit an den Inhalten der Szenarien}

Das Kernelement szenarienbasierter Softwareentwicklungsmethoden sind die sogenannten Szenarien und die ihnen zugrunde liegenden Geschichten - sie setzen die konkrete Situation der Techniknutzung mit all ihren auf die Nutzer_innen bezogenen Details in Szene und bilden damit die Basis für Designentscheidungen, die eben von den Nutzer_innen ausgehen und nicht von der „I-Methodology“ geprägt sein sollen. In den einschlägigen Lehrbüchern wird dieser Prozess der Datenerhebung, Geschichten- und Szenarienentwicklung sowie darauf gründender Technikgestaltung bislang indessen weder methodologisch noch epistemologisch problematisiert. So erscheinen Datenerhebungs- und Technikentwicklungsprozess als Vorgänge, in denen die „tatsächliche“ Techniknutzung unmittelbar und im Sinne einer direkt abfragbaren Information zu erheben, anschließend sachlich „objektiv“ darzustellen und umzusetzen ist. Theoretisch zugespitzt könnte man sagen, dass die erhobenen Geschichten mehr oder weniger als Abbild wirklicher Tätigkeitsabläufe gewertet und im Kontext der Szenarienarbeit nicht genauer hinterfragt werden. Betrachtet man diese Geschichten als Wissen und geht davon aus, dass dieses Wissen stets notwendigerweise eine soziale Konstruktion darstellt, so stellt sich die Frage, was Softwareentwickler_innen genau erfahren können, wenn sie diese Geschichten als Widerspiegelung der Realität behandeln. Wie gehen sie im Rahmen szenarienbasierter Softwareentwicklung mit Geschichten um, die inkohärent sind, Varianten enthalten, Widersprüche aufmachen oder in Sackgassen führen? Gerade dann sind diese Geschichten u. E. aufschlussreich für das Verständnis sozialer Welt, wenn sie nicht vorschnell vereinfacht und geglättet sind. Doch wer entscheidet dann auf welcher Grundlage, welcher Teil oder welche Variante der Geschichte der/die ,richtige“ ist und so der Softwaregestaltung zugrunde gelegt werden soll?

Damit Softwareentwicklung eine angemessene Basis für ihre Lösungsfindung erhalten kann, ist daher ein kritisch-analytischer Umgang mit Wissenskonstruktionen erforderlich. Denn Geschichten können inhaltlich ebenso bestehende Ungleichheitsverhältnisse, z. B. typische Frauenarbeit, reproduzieren wie sie implizit Vorstellungen über Geschlecht, Religion oder Gesundheit, z. B. über einen normalen leistungsfähigen Mann, verfestigen können. Kurz: Nur weil die Geschichten mit unserem Alltagswissen deckungsgleich sind, heißt das deshalb noch nicht, dass sie „die Realität“ der Nutzer_innen darstellen. Diese Geschichten bedürfen daher einer reflexiven Analyse, um die in ihnen beschriebenen Szenarien mit fachlichem, im 
Hinblick auf Geschlecht und Intersektionalität geschulten Blick zu deuten. In einer solchen Deutung wird dann danach gefragt, welche Konstruktionen von Geschlecht die Geschichte enthält und macht diese so als etwas kenntlich, das auch anders sein könnte.

Eine solche Umgangsweise mit den Inhalten der Szenarien birgt die Möglichkeit, Gestaltungsentscheidungen auf einer komplexeren Grundlage zu treffen. Sie bedenkt einerseits die Möglichkeiten und Grenzen von Erkenntnis mit, andererseits die Möglichkeit anderer Konstruktionen. So würde sie beispielsweise genauer darauf achten, welche Geschichten Nutzer_innen in welchem Zusammenhang denn überhaupt legitimerweise erzählen können und welche sie gegebenenfalls nicht erzählen. Sie würde auch der Option Aufmerksamkeit schenken, dass Nutzer_innen uneinheitliche Geschichten erzählen, die vielleicht auch miteinander in Konflikt stehen und so auf die Notwendigkeit hindeuten, dass unterschiedliche Deutungen der Realität existieren, die heterogenen Nutzer_innensichten entsprechen, und der eine Gestaltungslösung der HCI u. U. Rechnung tragen könnte. Schließt man an die eingangs beschriebene theoretische Perspektive an, so eröffnet sich die Möglichkeit, die Inhalte der Geschichten analytisch so zu bearbeiten, dass verkürzte Sichten auf Geschlecht und soziale Ungleichheitsverhältnisse offen gelegt und Geschichten in Zusammenarbeit mit den Nutzer_innen entworfen werden, die vielleicht gerade quer zum gängigen Alltagswissen liegen. Derartige alternative Geschichten könnten Gestaltungsspielräume öffnen, die nicht nur jenseits der „I-Methodology“, sondern auch jenseits verinnerlichter Geschlechtervorstellungen der Nutzer_innen selbst liegen. Vom ,track“ abweichende Geschichten, wie sie weiter oben diskutiert wurden, könnten vor dem Hintergrund der hier vorgeschlagenen methodologischepistemologischen Reflexion als produktives Moment der Gestaltung von HCI und damit als Chance für kreative Neugestaltungen angesehen werden.

\section{3}

\section{Ausblick: Offen partizipative, kooperative und nicht- hierarchische Szenarien-Arbeit}

In diesem Beitrag wurde exemplarisch der Bedarf und die Möglichkeit der Weiterentwicklung von vorhandener Methodik der Gestaltung von Human-Computer Interaction aus geschlechter- und intersektionalitätskritischer Perspektive diskutiert. Vorgeschlagen wurde eine methodische Weiterentwicklung durch am Prinzip der Offenheit orientierte, qualitative Erhebungsmethoden sowie die methodologisch-epistemologische Reflexion von Wissenskonstruktionen in und durch Szenarien. Beide Momente der Weiterentwicklung eröffnen die Chance, von der „I-Methodology“ abweichende und den alltäglichen Vorstellungen über Nutzer_innen widersprechende Perspektiven auf die Techniknutzung offen zu legen und für die Softwareentwicklung zugänglich $\mathrm{zu}$ machen. Um als derart produktive Elemente die Softwaregestaltungsmethoden $\mathrm{zu}$ bereichern bedarf es aber einer fundierten methodischen wie theoretischen Expertise. An dieser Stelle kann u. E. eine interdisziplinäre Zusammenarbeit von Softwareentwickler_innen und Geschlechterforscher_innen einsetzen.

Ganz im Sinne einer konsequenten Umsetzung szenarienbasierter Ansätze wäre die Arbeit an und mit Szenarien als integraler Bestandteil der Gestaltung von HCI zu verstehen, sodass Geschlechterforscher_innen ihre Expertise in der Erhebung der den Szenarien zugrundeliegenden Geschichten, dem Entwurf der Szenarien und der Umwandlung dieser in Softwarelösungen während des gesamten Softwareentwicklungsprozesses einbringen könnten. Ihre Aufgabe wäre, im Anschluss an die oben ausgeführten Weiterentwicklungspotentiale sowohl 
einen Beitrag zu einer offenen Erhebungsweise von Geschichten zu leisten als auch auf Grundlage ihrer Kenntnis der Geschlechter- und Intersektionalitätsforschung die analytische Arbeit mit den Inhalten der Szenarien zu unterstützen. Eine solche Form der interdisziplinären Zusammenarbeit als offen partizipative, kooperative, interdisziplinäre und nichthierarchische Szenarien-Arbeit hätte so das Potential, die mit der „I-Methodology“ verbundenen Engführungen zu vermeiden. Darüber hinaus birgt sie die Aussicht, Geschichten zu generieren, die zunächst nah an den Perspektiven der Befragten bleiben, diese im Folgeschritt auf Basis geschlechter- und intersektionalitätskritischen Fachwissens zu analysieren, den ihnen inhärenten sozialen Konstruktionen auf den Grund zu gehen und schließlich in den kooperativen Prozess des Konstruierens angemessener Szenarien oder Szenarienbündel einzutreten.

Versteht man Softwareentwicklung als Teil sozialer Konstruktionsprozesse von Geschlecht (und ihrer Interdependenz mit anderen sozialen Ungleichheitskategorien), so könnte die um die hier vorgeschlagenen Perspektiven erweiterte Gestaltung von HCI zu einem Moment gesellschaftlicher Veränderung werden und dazu beitragen, Ungleichheitsverhältnisse abzubauen. Vor allem aus intersektionaler Perspektive würde sichtbar werden können, dass es nicht immer nur die eine Geschichte gibt, sondern unterschiedliche und u. U. nicht immer kompatible Geschichten. So könnte auch die Vielfältigkeit der Nutzer_innen systematisch und produktiv in den Blick genommen werden und heterogene Interessensvertreter_innen gezielt in den Prozess der Gestaltung von HCI einbezogen werden. Das Ergebnis könnten vielleicht neue, multiperspektivische Gestaltungslösungen sein, die verschiedene Sichtweisen innerhalb einer Software anbieten und damit auf verschiedene Szenarien eingehen.

\section{Literatur}

Bath, C. (2009). De-Gendering informatischer Artefakte. Grundlagen einer kritisch-feministischen Technikgestaltung (Dissertation). Bremen: Staats- und Universitätsbibliothek Bremen. URN:

http://nbn-resolving.de/urn:nbn:de:gbv:46-00102741-12

Bath, C. (2007). „Discover Gender“ in Forschung und Technologieentwicklung? Soziale Technik 4, 35 .

Bath, C., Schelhowe, H. \& Wiesner, H. (2010). Informatik: Geschlechteraspekte einer technischen Disziplin. In R. Becker \& B. Kortendiek (Hrsg.), Handbuch Frauen- und Geschlechterforschung (S. 829-841). Wiesbaden: Springer.

Becker-Schmidt, R. (1987). Die doppelte Vergesellschaftung - die doppelte Unterdrückung: Besonderheiten der Frauenforschung in den Sozialwissenschaften. In L. Unterkirchner \& I. Wagner (Hrsg.), Die andere Hälfte der Gesellschaft (S. 10-25). Wien: Verlag des Österreichischen Gewerkschaftsbundes.

Beer, U. (1990). Geschlecht, Struktur, Geschichte. Soziale Konstituierung des Geschlechterverhältnisses. Frankfurt/M., New York: Campus.

Bowker, G. \& Leigh Star, S. (2000). Sorting Things Out. Classifications and its Consequences. Cambridge: MIT Press.

Buchmüller, S. (2013). Partizipation = Gleichberechtigung? Eine Betrachtung partizipativen Gestaltens im Kontext feministischer Designforschung und -praxis. In C. Mareis, M. Held \& G. Joost (Hrsg.), Wer gestaltet die Gestaltung? Praxis, Theorie und Geschichte des partizipatorischen Designs (119-139).

Bielefeld: transcript.

Crutzen, C. (2003). ICT-Representations as Transformative Critical Rooms. In G. Kreutzner \& H.

Schelhowe (Hrsg.), Agents of Change (S. 87-106). Opladen: Leske + Budrich. 
Ernst, W. \& Horwath, I. (Hrsg.) (2014a). Gender in Science and Technology. Interdisciplinary Approaches. Bielefeld: transcript.

Ernst, W. \& Horwath, I. (2014b). Introduction. In W. Ernst \& I. Horwath (Hrsg.), Gender in Science and Technology. Interdisciplinary Approaches (S. 7-15). Bielefeld: transcript.

Flick, U., Kardorff, E. von \& Steinke, I. (2007). Was ist qualitative Forschung? Einleitung und Überblick. In U. Flick, E.t von Kardorff \& I. Steinke (Hrsg.), Qualitative Forschung. Ein Handbuch (1329). Reinbeck bei Hamburg: Rowohlt.

Gildemeister, R. (2008). Doing Gender: Soziale Praktiken der Geschlechterunterscheidung. In R. Becker \& B. Kortendiek (Hrsg.), Handbuch Frauen- und Geschlechterforschung (S. 137-145). Wiesbaden: Springer.

Helduser, U., Marx, D., Paulitz, T. \& Pühl, K. (2004). Under construction? Konstruktivistische Perspektiven in feministischer Theorie und Forschungspraxis. Frankfurt/Main, New York: Campus.

Hoffmann-Riem, C. (1980). Die Sozialforschung einer interpretativen Soziologie. Der Datengewinn. Kölner Zeitschrift für Soziologie und Sozialpsychologie, 32, 339-372.

Hofmann, J. (1999). Writers, texts and writing acts: gendered user images in word processing software. In D. MacKenzie \& J. Wajcman (Hrsg.), The social shaping of technology (S. 222-243). London, New York: Open University Press.

John, S. (2006). Un/realistically embodied: The gendered conceptions of realistic game design. Online Proceedings of 2006 AVI Workshop „Gender and Interaction. Real and Virtual women in a male world", Venice, Italy.

Kerner, I. (2009). Differenzen und Macht. Zur Anatomie von Rassismus und Sexismus. Frankfurt/M., New York: Campus.

Knapp, G.-A. (1990). Zur widersprüchlichen Vergesellschaftung von Frauen. In E.-H. Hoff (Hrsg.), Die doppelte Sozialisation Erwachsener (S. 17-52). München: DJI Verlag.

Lucht, P. \& Paulitz, T. (2008). Recodierungen des Wissens. Stand und Perspektiven der Geschlechterforschung in Naturwissenschaften und Technik. Frankfurt/Main, New York: Campus.

Lübke, V. (2005). CyberGender. Geschlecht und Körper im Internet. Königstein, Taunus: Ulrike Helmer Verlag.

McGraw, K. \& Harbison, K. (1997). User-Centered Requirements. The Scenario-Based Engineering Process. Mahwah, New Jersey: Lawrence Erlbaum.

Paulitz, T. (2008). Technikwissenschaften: Geschlecht in Strukturen, Praxen und Wissensformationen der Ingenieurdisziplinen und technischen Fachkulturen. In R. Becker \& B. Kortendiek (Hrsg.), Handbuch Frauen- und Geschlechterforschung (S. 779-790). Wiesbaden: Springer.

Rommes, E. (2014). Feminist Interventions in the Design Process. In W. Ernst \& I. Horwath (Hrsg.), Gender in Science and Technology. Interdisciplinary Approaches (S. 41-55). Bielefeld: transcript.

Rommes, E. (2002). Gender Scripts and the Internet: The Design and Use of Amsterdam's Digital City. University of Twente, Enschede.

Rommes, E., Van Oost, E. \& Oudshoorn, N. (1999). Gender in the Design of the Digital city of Amsterdam. Information, Communication \& Society, 2(4), 476-495.

Rosson, M. B. \& Carroll, J. M. (2002). Usability Engineering. Scenario-Based Development of Human-Computer Interaction. San Francisco: Morgan Kaufmann.

Schelhowe, H. (2006). Informatik. In C. von Braun \& I. Stephan (Hrsg.), Gender-Studien. Eine Einführung (S. 201-210). Stuttgart, Weimar: Metzler Verlag. 
Schiebinger, L., Klinge, I., Sánchez de Madariaga, I., Schraudner, M. \& Stefanick, M. (2011-2013). Gendered Innovations in Science, Health \& Medicine, Engineering, and Environment. URN: http://genderedinnovations.stanford.edu/index.html.

Singer, M. (2005). Geteilte Wahrheit. Feministische Epistemologie, Wissenssoziologie und Cultural Studies. Wien: Löcker Verlag.

Smykalla, S. \& Vinz, D. (2013). Intersektionalität zwischen Gender und Diversity. Theorien, Methoden und Politiken der Chancengleichheit. Münster: Westfälisches Dampfboot.

Wetterer, A. (2008). Konstruktion von Geschlecht: Reproduktionsweisen der Zweigeschlechtlichkeit. In R. Becker \& B. Kortendiek (Hrsg.), Handbuch Frauen- und Geschlechterforschung (S. 122-131). Wiesbaden: Springer.

Winker, G. \& Degele, N. (2009). Intersektionalität. Zur Analyse sozialer Ungleichheiten. Bielefeld: transcript. 
